\title{
Vibrations of a pipe on elastic foundations
}

\author{
S V LILKOVA-MARKOVA and D S LOLOV \\ Department of Technical Mechanics, Faculty of Hydro-technics, University of \\ Architecture, Civil Engineering \& Geodesy, Sofia 1046, Bulgaria \\ e-mail: lilkova_fhe@uacg.bg
}

MS received 28 July 2003; revised 28 January 2004

\begin{abstract}
A cantilevered pipe subjected to external transverse (or lateral) force is investigated. Two cases of elastic foundations are considered: rotational and both linear and rotational. The major findings are the variations in frequency with flow velocity and displacements at different points and times.
\end{abstract}

Keywords. Cantilevered pipe; vibrations of pipes; elastic foundations; external transverse force.

\section{Introduction}

The dynamic stability of pipes is a broad area of investigation. Elishakoff \& Impollonia (2001) carried out research on cantilevered pipes mounted on elastic Winkler and rotary foundations. Both elastic foundations are partially attached to the pipe. These foundations influence the critical velocity of the fluid. Becker et al (1978) studied the effect of the rotational Pasternak elastic foundation with a constant $c_{2}$, defined as the rigidity of the distributed elastic rotational springs at the length of the pipe. A new term appears in the equation of the transverse vibrations. This new term is opposed to the centrifugal term $U^{2} w_{, x x}$ and has a strong effect on the stability. Lilkova-Markova \& Lolov (2003) investigated the influence of the transverse force at the free end of the dynamic stability of a cantilevered pipe placed on a Winkler elastic foundation.

In this paper, two cases of cantilevered pipes with transverse (or lateral) force at the free end are investigated, such as, (a) a pipe on a rotational Pasternak elastic foundation, and (b) a pipe on a translational and rotational elastic foundation.

\section{Model}

Forces due to gravitation, internal damping, external applied compression at the axis of the pipe and viscous damping due to friction between the pipe and the fluid, are neglected in this analysis.

The differential equation describing the local movement of the arbitrary point on the pipe is given as:

$$
K w_{, x x x x}+\left(M U^{2}-c_{2}\right) w_{, x x}+2 M U w_{, x t}+(m+M) w_{, t t}+c_{1} w=F(x, t),
$$


where $K$ is the bending rigidity of the pipe, $M$ is the mass of the fluid per unit length, $m$ is the mass of the pipe per unit length, $U$ is the velocity of the flowing fluid, and $c_{1}$ is the Winkler constant of the elastic foundation. The Winkler constant $c_{1}$ has a sense of a force due to unit vertical displacement. The constant proportionality coefficient $c_{2}$ is constant for the Pasternak elastic foundation, and is a local foundation response bending moment due to local unit rotation of the pipe.

The decision of the problem was made by the method of Galerkin. The function of vertical displacement is in the following form:

$$
w(x, t)=\sum_{i=1}^{n} Y_{i}(x) q_{i}(t) .
$$

The basic basis of functions $Y_{1}(x)$ consists of mode shapes of the pipe when the fluid is stationary. They are obtained for a cantilevered pipe on the type of the elastic foundation considered.

Substituting (2) in (1) and following transformations after Wu \& Shin (2001), the following equation for the associated generalized co-ordinates is obtained:

$$
[\bar{M}]\{q \dot{q}(t)\}+[\bar{C}]\{q(\dot{t})\}+[\bar{K}]\{q(t)\}=\{\bar{F}(t)\},
$$

where $\bar{M}[n, n]$ is the diagonal matrix with elements equal to the sum of the mass of the fluid and mass of the pipe per unit length. The elements of the matrix $\bar{C}[n, n]$ and $\bar{K}[n, n]$ are obtained from formulae given in $\mathrm{Wu} \&$ Shin (2001). For them the mode shapes for the pipe with stationary fluid are used. The elements in $\bar{F}(t)$ reflect the existence of a transverse force at the free end of the pipe. This force is dependent on the time $t$. For the numerical investigating, the force $F(t)=100 t$. The influence of Pasternak's elastic foundation with different constants is studied here.

\section{Results and discussion}

The cantilevered pipe of $6 \mathrm{~m}$ length is considered in the numerical investigation. The typical points on the pipe are $1 \mathrm{~m}$ interval. The inside diameter of the cross section of the pipe is $0.19 \mathrm{~m}$ and outer diameter $-0.20 \mathrm{~m}$. The bending rigidity $K$ of the steel pipe is $3001.008 \mathrm{KNm}^{2}$.

The values of the circular frequencies of the pipe are obtained for different values of the flow velocity as given $\mathrm{Wu} \&$ Shin (2001). In figure 1, the first six of them are shown when the pipe is lying only on an Pasternak elastic foundation with a constant $c_{2}=200 \mathrm{kNm} / \mathrm{m}$. It is evident that the flow velocity is affected more for the second and the third frequencies.

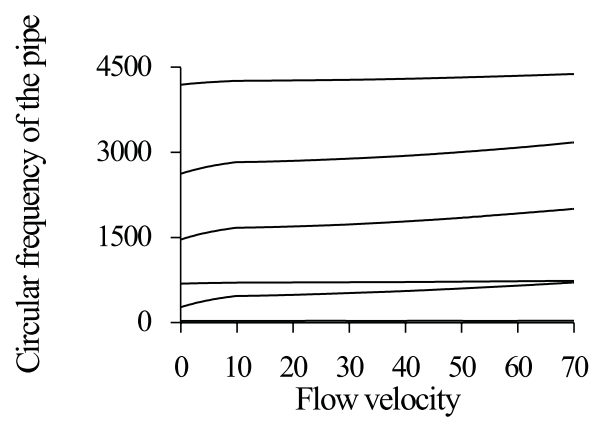

Figure 1. The case of the Pasternak foundation. 


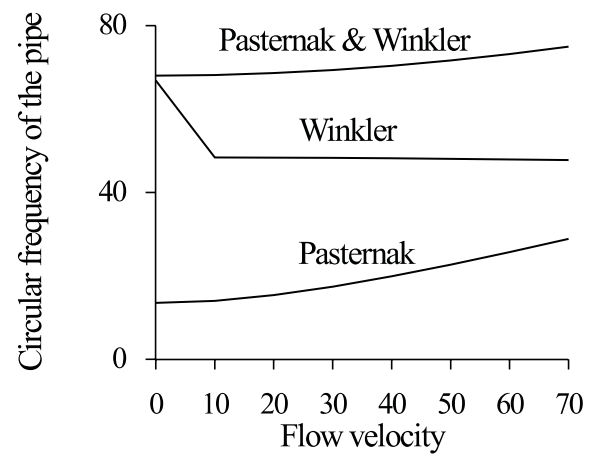

Figure 2. Three cases of elastic foundation.

The first circular frequency is calculated and in two other cases, only a Winkler foundation with constant $c_{1}=500 \mathrm{kN} / \mathrm{m}$ and the same Winkler and Pasternak foundation with constant $c_{2}=200 \mathrm{kN} / \mathrm{m}$, simultaneously. The dependence of the flow velocity is presented in figure 2 . In the case of both the elastic foundations, the circular frequencies are maximum, but when the pipe is on a Pasternak elastic foundation they are feeble.

The influence of the constant $c_{2}$ of the Pasternak elastic foundation on the vertical displacements of the points on the axis of the pipe is investigated. The values of $c_{2}$ considered are 100, 200 and $300 \mathrm{kNm} / \mathrm{m}$. Winkler elastic foundation constant in these three cases is $c_{1}=500 \mathrm{kN} / \mathrm{m}$. The flow velocity is equal to $20 \mathrm{~m} / \mathrm{s}$. The vertical dynamical displacements in the typical points of the pipes for $t=0,1,2,3,4,5$ are shown in figures $3 \mathrm{a}-\mathrm{c}$. It is evident that for values of $c_{2}$ equal to 200 and $300 \mathrm{kNm} / \mathrm{m}$, the displacements are very close.

(a)

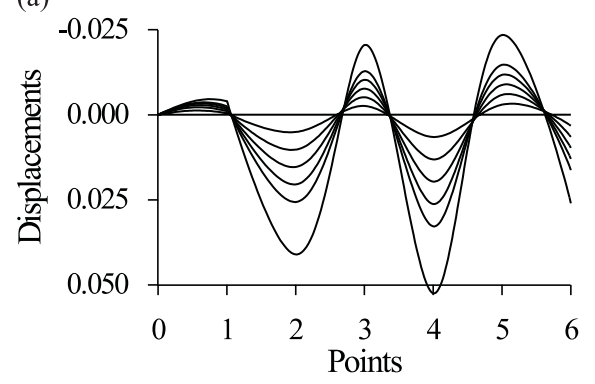

(c)

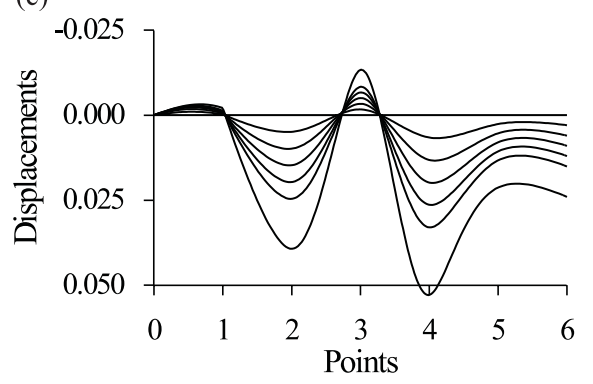

(b)

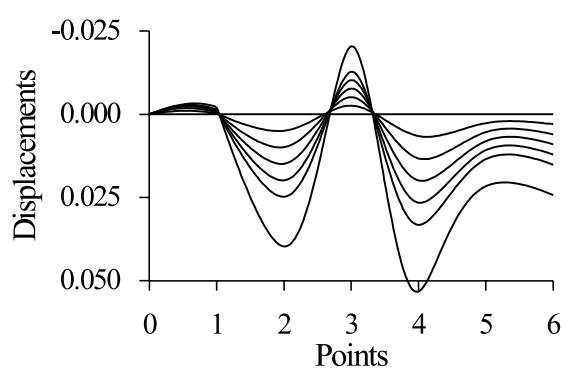

Figure 3. $\quad c_{1}=500 ; c_{2}=100(\mathbf{a}), 200$ (b) and $300(\mathbf{c})$. 


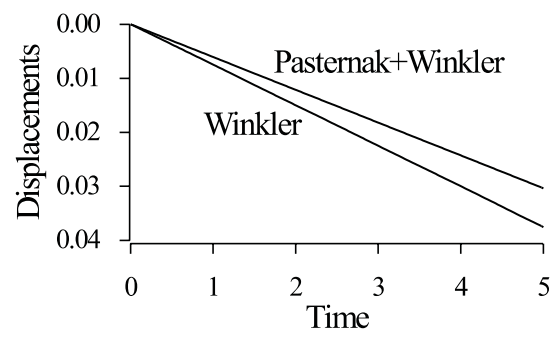

Figure 4. Displacements as the free end of the pipe in the case where $c_{1}=500 ; c_{2}=200$.

Displacements at the free ends of the cantilevered pipes in the case where $c_{1}=500 \mathrm{kN} / \mathrm{m}$ and $c_{2}=200 \mathrm{kNm} / \mathrm{m}$ depending on time are shown in figure 4 . They increase with time due to the transverse force at the free end. The presence of the Pasternak and Winkler elastic foundation has the expected influence.

\section{Conclusions}

Pasternak elastic foundation combined with Winkler elastic foundation has a considerable effect on the circular frequencies of pipes conveying fluids and on the vertical transverse displacements at different points and times.

\section{References}

Becker M, Hauger W, Winzen W 1978 Exact stability analysis of uniform cantilevered pipes conveying fluid or gas. Arch. Mech. (Polish Acad. Sci.) 30: 757-768

Elishakoff I, Impollonia N 2001 Does a partial elastic foundation increase the flutter velocity of a pipe conveying fluid. J. Appl. Mech. 68: 206-212

Lilkova-Markova S V, Lolov D S 2003 Cantilevered pipe conveying fluid, lying on Winkler elastic foundation and loaded by transversal force at the free end. J. Building (Sofia) (4): 5-8

Wu J-S, Shin P-Y 2001 The dynamic analysis of a multispan fluid-conveying pipe subjected to external load. J. Sound Vibr. 239: 201-215 